\title{
Tratamiento del carcinoma basocelular periocular con una combinación sinérgica de interferones alpha-2b y gamma
}

\section{Synergistic effect of combined IFN-alpha2b and IFN-gamma treatment for periocular basal cell carcinoma}

\author{
Yairan Negrín-Caceres ${ }^{1 *}$, Ailyn C. Cabrera-Romero ${ }^{1}$, Ledisleydy Cárdenas-Monzón ${ }^{1}$, \\ Arletis Ferrer-Pérez ${ }^{2}$ y Norma E. Batista-Hernández ${ }^{3}$ \\ ${ }^{1}$ Centro Oftalmológico, Hospital Arnaldo Milián Castro; ${ }^{2}$ Hospital Arnaldo Milián Castro; ${ }^{3}$ Unidad de Investigaciones Biomédicas, Universidad de \\ Ciencias Médicas. Villa Clara, Cuba
}

\section{Resumen}

Objetivo: Evaluar los resultados de la aplicación del HeberPAG ${ }^{\oplus}$ perilesional, en carcinoma basocelular periocular. Método: Se realizó una investigación experimental, en un grupo de siete pacientes a los que se les aplicó un mismo esquema de tratamiento con HeberPAG ${ }^{\oplus} 10.5 \times 10^{6}$ UI perilesional 3 veces a la semana durante 3 semanas consecutivas. Se consideraron variables demográficas, así como tiempo de evolución, tamaño inicial de la lesión, subtipo clínico, subtipo histológico, respuesta clínica, respuesta objetiva (RO) y eventos adversos. Resultados: El tiempo promedio de evolución de la lesión fue de $7.0 \pm 1.6$ meses, con un diámetro mayor medio de $17.4 \pm 2.3 \mathrm{~mm}$. El subtipo clínico nódulo ulcerativo se presentó en el $42.9 \%$ de los casos y los subtipos histológicos sólido y superficial se presentaron con igual proporción, en un $42.9 \%$. El diámetro mayor de la lesión mostró un comportamiento decreciente en función del tiempo, y a las 16 semanas se alcanzó una respuesta clínica completa y objetiva en el $85.7 \%$ de los casos. Todos los pacientes presentaron eventos adversos, que se resolvieron con medicación oral sin abandonar el tratamiento. La totalidad de ellos ofrecieron un alto grado de satisfacción con el tratamiento. Conclusiones: El empleo del HeberPAG ${ }^{\circledR}$ resultó ser efectivo, por lo que se podría considerar como una alternativa útil y segura como tratamiento conservador en pacientes con carcinoma basocelular periocular cuando otras terapias no son posibles.

Palabras clave: Carcinoma basocelular. Interferones. HeberPAG ${ }^{\oplus}$.

\section{Abstract}

Purpose: To evaluate the results for the application of the perilesional HerberPAG on periocular basal cell carcinoma. Methods: An experimental investigation was carried out on a group of 7 patients who were given the same treatment regimen with HeberPAG ${ }^{\odot} 10.5 \times 106$ UI 3 times a week for 3 consecutive weeks. Demographic variables where considered as well as time of evolution, initial injury size, clinical subtype, histological subtype, clinical response, objective response and adverse effects. Results: The average time of evolution of the injury was $7.0 \pm 1.6$ months, with a higher diameter medium of $17.4 \pm 2.3 \mathrm{~mm}$. The clinic nodule-ulcerative subtype was presented on $42.9 \%$ of the subjects and the solid and superficial

\section{Correspondencia:}

*Yairan Negrin-Caceres

Av. Nuevo Hospital e/Doble Vía y Circunvalación Fecha de recepción: 07-06-2017

C.P. 50100, Santa Clara, Villa Clara, Cuba Fecha de aceptación: 01-03-2018

E-mail: ynegrinc@gmail.com

DOI: 10.24875/RMO.M18000029
Disponible en internet: 14-05-2018 Rev Mex Oftalmol. 2018;92(3):136-143 www.rmo.com.mx 0187-4519/○ 2018 Sociedad Mexicana de Oftalmología. Publicado por Permanyer México. Este es un artículo Open Access bajo la licencia CC BY-NC-ND (http://creativecommons.org/licenses/by-nc-nd/4.0/). 
hostiological subtypes were presented with an equal proportion of $42.9 \%$. Conclusions: The use of HerberPAG was effective and could be considered as a useful and a safe alternative as a conservative treatment in subjects with periocular basal cell carcinoma when other therapies are not available.

Key words: Basal cell carcinoma. Interferons. HeberPAG ${ }^{\oplus}$.

\section{Introducción}

El carcinoma basocelular (CBC) es una neoplasia maligna derivada de las células no queratinizadas que se originan de la capa basal de la epidermis. Es el cáncer más común en los humanos ${ }^{1,2}$.Existen 2.8 millones de casos diagnosticados de CBC cada año en EE.UU. y 700,000 nuevos casos en Europa. El cáncer de piel en la población estadounidense supera las estadísticas de otros tipos de cáncer, está estimado que 1 de cada 5 norteamericanos desarrollará cáncer de piel durante su vida ${ }^{3,4}$. Representa entre un 80 a $90 \%$ de las afecciones malignas de la piel ${ }^{5}$. A su vez, es el tumor maligno periocular más frecuente $(80-95 \%)^{6,7}$. Su etiopatogenia está relacionada con la edad, factores genéticos, virales y ambientales (radiación ultravioleta) ${ }^{3}$. En la última década se ha detectado un incremento de su incidencia y una tendencia a aparecer en edades más tempranas ${ }^{5}$.

Inicialmente se presenta como un tumor pequeño de crecimiento lento, como una lesión ulcerada, con telangiectasias y presencia de un borde redondeado; sin embargo, sus signos pueden variar de acuerdo al subtipo clínico. La destrucción local que provoca puede ser significativa en estructuras importantes como los párpados, y puede generar deformidades o pérdida de la función del órgano afectado 7,8 .

Elegir el tratamiento apropiado dependerá en cada caso del tamaño de la lesión, localización, subtipo de CBC, estado general, edad y necesidades estéticas del paciente. La cirugía continúa siendo la primera opción terapéutica. Tradicionalmente, el $\mathrm{CBC}$ ha sido completamente removido con 3 a $4 \mathrm{~mm}$ de margen quirúrgico combinado con reconstrucción primaria, y se han obtenido excelentes resultados ${ }^{9}$. Alternativas como radioterapia, crioterapia, ablación con láser, quimioterapia, inmunoterapia han sido descritas como variantes útiles en casos inoperables o lesiones extensas. El tratamiento tópico con Imiquimod puede ser una alternativa a la cirugía, pero el resultado a largo plazo no es tan exitoso como otras modalidades ${ }^{10}$. La terapia fotodinámica generalmente no se recomienda para el manejo del CBC debido a las bajas tasas de éxito en cabeza y cuello ${ }^{11-13}$. El tratamiento conservador del $\mathrm{CBC}$ cobra importancia en la región periocular, pues, cuando la cirugía se realiza respetando el margen oncológico a este nivel, puede implicar la extirpación de amplias áreas importantes del aparato de la visión. La reconstrucción de la zona afectada puede causar alteraciones estético-funcionales graves, además, por razones estéticas, existe la tendencia a que en la cirugía de la cara se elimine la menor cantidad de tejido posible y los bordes de los tumores no sean bien resecados, lo que aumenta considerablemente el riesgo de recidivas ${ }^{9,14,15}$. Una valiosa herramienta en el tratamiento conservador del CBC constituye la inmunoterapia ${ }^{16}$. Las inyecciones intralesionales de interferón (IFN) fueron reportadas como efectivas para el tratamiento del CBC en un estudio preliminar en 1986. Otros estudios coinciden en que el uso de IFN en el tratamiento del CBC y del carcinoma de células escamosas (CCE) ha mostrado una amplia gama de respuesta $(60-100 \%)$ con una tasa de recurrencia de hasta un $17 \%{ }^{17}$.

Aunque la bibliografía sobre este tópico no es amplia, ya se han publicado alentadores resultados sobre la administración de la combinación sinérgica de IFN alfa-2b e interferón gamma (HeberPAG ${ }^{\circledR}$, Heber Biotec SA, La Habana, Cuba), registrada en el año 2008 por el Centro para el Control Estatal de la Calidad de los Medicamentos [CEDMED], autoridad reguladora de medicamentos en Cuba ${ }^{17}$, indicada en el tratamiento de todo subtipo de $\mathrm{CBC}$ mediante inyección intratumoral y/o perilesional en lesiones cutáneas de CBC, generando regresión total de las mismas ${ }^{18-20}$. Esta alternativa potencia la farmacocinética del fármaco por la combinación de dos principios activos que pueden actuar sinérgicamente. De esta forma se obtienen las mismas ventajas con un nuevo mecanismo de distribución que incluye un incremento y prolongación de la actividad farmacológica sin toxicidad adicional, disminución en la frecuencia de inyecciones, junto a una mayor conformidad y calidad de vida del paciente ${ }^{21}$.

Es objetivo de los autores de esta investigación evaluar los resultados de la aplicación del HeberPAG ${ }^{\circledR}$ perilesional, en el CBC periocular en la serie de pacientes tratados. 


\section{Metodología}

Se realizó un estudio experimental (ensayo clínico de fase IV), prospectivo, en pacientes con diagnóstico de CBC periocular, atendidos en la consulta de oculoplastia en el Hospital Universitario Arnaldo Milián Castro de Villa Clara, Cuba, en el periodo comprendido entre febrero del año 2014 hasta febrero del 2015.

Se incluyeron 7 pacientes de ambos sexos, mayores de 18 años, con diagnóstico de CBC periocular en cualquier estadio y/o subtipo clínico, cuya lesión fuera mayor de $1 \mathrm{~cm}$ en la cual no estuviera recomendado el tratamiento quirúrgico y lesiones con exéresis incompleta con tratamiento quirúrgico previo o recidivantes. Se excluyó a mujeres embarazadas, pacientes que en el momento de la inclusión estuvieran recibiendo tratamientos oncoespecíficos y pacientes con hipersensibilidad a los IFN.

El diagnóstico positivo fue establecido mediante las características clínicas de la lesión, resultados histológicos y dermatoscópicos. La biopsia incisional se realizó mediante la técnica de punch de $4 \mathrm{~mm}$, fijando el tejido en una solución de formalina al $5 \%$ y procediendo a su inclusión en parafina para observar sus cortes en un microscopio con luz convencional. La dermatoscopia fue realizada por un especialista en Dermatología, con este examen se evidenció la presencia o ausencia de signos sugestivos de tumor. Ambos estudios se practicaron al inicio y a las 16 semanas después del tratamiento. A todos los pacientes de la serie se les aplicó un mismo esquema de tratamiento, con la formulación farmacéutica estabilizada que contenía una combinación sinérgica de IFN alfa $2 b$ y gamma, fosfato hidrogenado de sodio, dextrano 40 , cloruro de sodio, y albúmina humana $\left(\right.$ HeberPAG ${ }^{\circledR}$, Heber Biotec $\mathrm{SA}$, Habana, Cuba) a una dosis de $\left.10.5 \times 10^{6} \mathrm{UI}\right) / 1 \mathrm{ml}$, mediante infiltración perilesional 3 veces a la semana durante 3 semanas consecutivas. Todos fueron tratados de forma ambulatoria y reevaluados en consulta a las $4,8,12$ y 16 semanas después de iniciado el tratamiento, donde se precisó el tamaño de la lesión, cambios clínicos y reacciones adversas.

Se consideraron variables demográficas, así como tiempo de evolución, tamaño inicial de la lesión, subtipo clínico, subtipo histológico, respuesta clínica, RO y eventos adversos.

El tamaño inicial de la lesión fue medido antes del tratamiento empleando una regla milimetrada, el subtipo histológico se clasificó según el informe anatomopatológico. La respuesta clínica se determinó según las características de la lesión a las 16 semanas de iniciado el tratamiento. Teniendo en cuenta este criterio se obtuvieron las siguientes categorías:

- Respuesta completa (RC): desaparición total de la lesión.

- Respuesta parcial (RP): reducción de al menos el $30 \%$ de la suma de los diámetros mayores, tomando como referencia la suma de los diámetros mayores de base.

- Respuesta estable (RE): reducción no suficiente para calificar como respuesta parcial.

- La RO se expresó por la suma de la RC + RP.

Se realizaron determinaciones hematológicas y bioquímicas (hemoglobina, hematocrito, recuento de leucocitos y plaquetas, transaminasa, creatinina, urea) antes y después del tratamiento.

El seguimiento a largo plazo continúa trimestralmente durante el primer año, con carácter semestral durante el segundo y el tercer año, y anual desde el cuarto hasta el décimo año.

La información de cada paciente fue registrada en un cuaderno de recogida de datos y se procesó mediante el paquete estadístico SPSS 20.0. Los resultados se presentaron en tablas y gráficos. Se aplicaron técnicas estadísticas según tipos de variables. Se estimaron intervalos de confianza bayesianos para proporciones para la respuesta clínica, trabajando con una confiabilidad del $95 \%$. Se cumplieron los principios éticos definidos en la declaración de Helsinki para experimentación en humanos y se obtuvo por escrito el consentimiento informado de cada paciente. Este estudio se realizó con la aprobación del comité de ética de la institución. El ensayo clínico se encuentra registrado en Registro Público Cubano de Ensayos Clínicos.

\section{Resultados}

En el período descrito fueron estudiados un total de 7 pacientes a quienes se les aplicó el tratamiento. Presentaron una edad media de 75 años (Tabla 1).

El tiempo medio de evolución del tumor fue de 7 meses y su diámetro mayor tuvo un valor promedio de $17.4 \mathrm{~mm}$. Predominó el subtipo clínico nódulo ulcerativo en el $42.9 \%$ de los casos, y el resto de los subtipos se presentaron en igual proporción, representando el $14,3 \%$ para cada caso. Desde el punto de vista histológico incidieron de igual forma los subtipos superficial y sólido afectaron a 3 pacientes, respectivamente, y solo 1 presentó subtipo infiltrante (Tabla 2).

En la serie estudiada se observó que en general hubo un comportamiento decreciente, regular y sostenido del 
Tabla 1. Características demográficas de la serie

\begin{tabular}{|l|c|}
\hline $\begin{array}{l}\text { Edad } \\
\text { Media } \pm \text { DE } \\
\text { Mín/Máx }\end{array}$ & $74.7 \pm 11.9$ \\
\hline & $54 / 89$ \\
\hline $\begin{array}{l}\text { Sexo (N. } \% \%) \\
\text { Masculino }\end{array}$ & \\
\hline Femenino & $3 / 42.9$ \\
\hline $\begin{array}{l}\text { Tipo de piel según escala de Fitzpatrick (N. } \% \%) \\
\quad \text { Tipo II } \\
\text { Tipo III }\end{array}$ & $4 / 57.1$ \\
\hline
\end{tabular}

Tabla 2. Evaluación inicial de la lesión

\begin{tabular}{|c|c|}
\hline $\begin{array}{l}\text { Tiempo de evolución (meses) } \\
\text { Media } \pm \text { DE } \\
\text { Mínimo } \\
\text { Máximo }\end{array}$ & $\begin{array}{c}7.0 \pm 1.6 \\
5 \\
9\end{array}$ \\
\hline $\begin{array}{l}\text { Diámetro mayor }(\mathrm{mm}) \\
\text { Media } \pm \mathrm{DE} \\
\text { Mínimo } \\
\text { Máximo }\end{array}$ & $\begin{array}{c}17.4 \pm 2.3 \\
15 \\
23\end{array}$ \\
\hline $\begin{array}{l}\text { Subtipo clínico (N.\%\%) } \\
\text { Nodular } \\
\text { Ulcerado } \\
\text { Nódulo ulcerativo } \\
\text { Pigmentado } \\
\text { Plano cicatricial }\end{array}$ & $\begin{array}{l}1 / 14.3 \\
1 / 14.3 \\
3 / 42.9 \\
1 / 14.3 \\
1 / 14.3\end{array}$ \\
\hline $\begin{array}{l}\text { Subtipo histológico (N.\%\%) } \\
\text { Infiltrante } \\
\text { Superficial } \\
\text { Sólido }\end{array}$ & $\begin{array}{l}1 / 14.3 \\
3 / 42.9 \\
3 / 42.9\end{array}$ \\
\hline
\end{tabular}

Tabla 3. Evaluación final de la lesión

$\begin{array}{lc}\begin{array}{l}\text { Respuesta clínica }\left[\mathrm{N} .{ }^{\circ}(\%)\right] \\ \text { Completa } \\ \text { Parcial }\end{array} & 6(85.7) \\ \text { Estable } & 0 \\ \text { Respuesta histológica }\left[\mathrm{N} .{ }^{\circ}(\%)\right] & 1(14.3) \\ \text { Sí } & \\ \text { No } & 6(85.7) \\ \text { Respuesta dermatoscópica }\left[\mathrm{N} .{ }^{\circ}(\%)\right] & 1(14.3) \\ \text { Sí } & \\ \text { No } & 6(85.7) \\ \text { Respuesta objetiva }\left[\mathrm{N} .{ }^{\circ}(\%)\right] & 1(14.3) \\ \text { Completa + parcial } & \end{array}$

diámetro mayor de la lesión en función del tiempo (Fig. 1).

En el $85.7 \%$ de los casos se obtuvo una RC al tratamiento, solo un paciente presentó respuesta estable, lo que representa el $\mathbf{1 4 . 3 \%}$. Estos valores mostraron igual comportamiento en el caso de la respuesta histológica y dermatoscópica (Tabla 3).
Tabla 4. Eventos adversos

\begin{tabular}{|c|c|c|c|}
\hline \multicolumn{2}{|c|}{ Evento adverso } & Frecuencia & $(\%)$ \\
\hline \multicolumn{2}{|c|}{ Eritema perilesional } & 7 & 100.0 \\
\hline \multicolumn{2}{|l|}{ Fiebre } & 5 & 71.4 \\
\hline \multicolumn{2}{|c|}{ Escalofríos } & 4 & 57.1 \\
\hline \multicolumn{2}{|c|}{ Astenia } & 4 & 57.1 \\
\hline \multicolumn{2}{|c|}{ Anorexia } & 4 & 57.1 \\
\hline \multicolumn{2}{|c|}{ Náuseas } & 1 & 14.3 \\
\hline \multicolumn{2}{|c|}{ Vómitos } & 1 & 14.3 \\
\hline \multicolumn{2}{|c|}{ Dolor muscular } & 1 & 14.3 \\
\hline \multicolumn{4}{|l|}{ 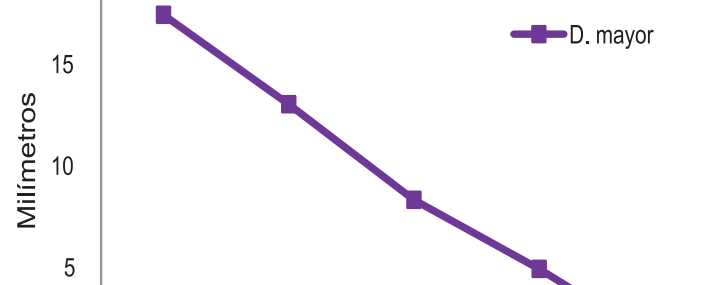 } \\
\hline 0 & Semana 1 & emana 8 Sema & mana 16 \\
\hline
\end{tabular}

Figura 1. Variación de la media del diámetro mayor.

Todos los pacientes estudiados presentaron eventos adversos relacionados con la administración del medicamento. El más frecuente fue el eritema perilesional, que se presentó en el $100 \%$ de los casos, y fiebre, en el $71.4 \%$ de ellos (Tabla 4).

\section{Discusión}

EI CBC periocular continúa encabezando la lista de tumores oculares a escala mundial ${ }^{9,22}$.Su manejo depende de factores relacionados con el paciente y con las características del tumor. La resección quirúrgica y reconstrucción continúa siendo el principal pilar del tratamiento; sin embargo, en algunas condiciones se convierte en una opción a justipreciar. La reconstrucción en la región periocular puede generar retracción palpebral, ectropión o entropión cicatricial, ptosis, ojo seco, recurrencia del tumor, triquiasis, infecciones, rechazo del injerto, cicatrices antiestéticas, hiper o hipopigmentación ${ }^{5,23}$. Actualmente hay autores que muestran un interés creciente en el uso de inmunoterapias como 
opción no quirúrgica en el tratamiento del cáncer de piel no melanoma. Como punto de partida de ello comienzan a registrarse resultados de la aplicación de combinaciones sinérgicas de IFN, que reportan invaluables ventajas.

En este estudio el grupo de pacientes tratados fueron personas que se encontraban en su séptima década de vida como promedio. Algunos artículos señalan que los procesos oncoproliferativos son más frecuentes en edades avanzadas, aunque recientemente se ha descrito que existe una tendencia creciente a que estas entidades aparezcan cada vez más frecuentemente en mujeres jóvenes ${ }^{8,24,25}$, posiblemente asociada a excesivos bronceados de piel y baños de sol26-29.

La mayoría de los pacientes presentó piel de fototipo II, según la clasificación internacional de Fitzpatrick, lo que coincide con la literatura, donde se describe que la piel blanca constituye un factor de riesgo para el desarrollo de $\mathrm{CBC}^{5,26}$,principalmente con el fenotipo cutáneo I, con pecas, ya sea con cabello rojo o rubio, y ojos claros. La historia de exposición a radiación solar, particularmente la radiación ultravioleta, en correlación inversa a la disminución de la pigmentación en la piel, es generalmente considerada como el principal factor de riesgo para el $\mathrm{CBC}^{26,30}$.

En el grupo estudiado no hubo diferencias significativas en cuanto al sexo, aunque hay autores que plantean que su presentación es más frecuente en el sexo masculino ${ }^{8,26}$, con una proporción aproximada de 1.5-2:124.

El diámetro medio de las lesiones diagnosticadas fue de $17.4 \mathrm{~mm}$. Se plantea que la metástasis por CBC es extremadamente rara ${ }^{17,31}$; sin embargo, existe un riesgo del $0.55 \%$ después de años de diagnosticado sin tratamiento. La metástasis toma ganglios linfáticos regionales, huesos, hígado y pulmón, principalmente cuando la lesión tiene un diámetro $>2 \mathrm{~cm}$, la resección es incompleta y tiene compromiso perineural $y / 0$ perivascular ${ }^{17,26}$.

En este estudio hubo un paciente al que se le diagnosticó un CBC periocular con diámetro de $2.3 \mathrm{~cm}$; sin embargo, tuvo una excelente respuesta al tratamiento con evolución clínica y estética sorprendente.

Con el tratamiento de la combinación sinérgica de IFN se obtuvo una RO en el $85.7 \%$ de los casos (RC $85.7 \%$ + RP 0), en cada caso la RC completa coincidió con el resultado histológico y dermatoscópico. Solo un paciente presentó $\mathrm{RE}$, ya que su lesión sufrió modificaciones sin llegar a un 30\% de reducción, y fue sometido a cirugía practicándose excéresis con amplio margen y reconstrucción estética. La RO obtenida en

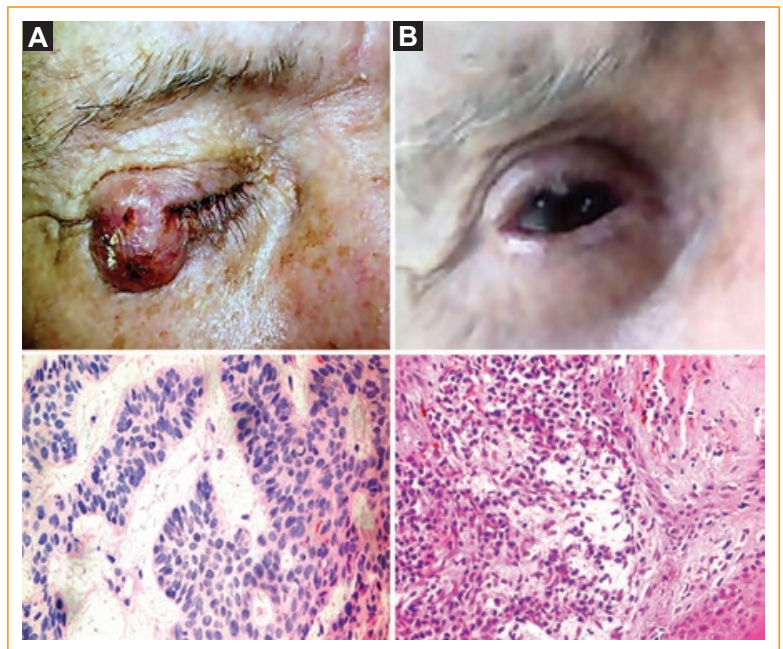

Figura 2. Paciente de 86 años con CBC de $2.3 \mathrm{~cm}$ de diámetro que ocupa el canto externo y $1 / 3$ externo de párpados superior e inferior, se obtuvo respuesta completa. En este caso, un posible tratamiento quirúrgico respetando los bordes de sección quirúrgica recomendados quizá no ofreciera los resultados estéticos obtenidos. A: antes del tratamiento; B: después del tratamiento.

estos casos fue similar a la descrita por García Vega, et al. ${ }^{19}$ en un estudio reciente sobre aplicación del HeberPAG ${ }^{\circledR}$ en la región periocular usando la misma dosis empleada en este estudio.

En las figuras 2, 3 y 4 se muestran ejemplos de RC y la figura 5 muestra un caso de RE. En cada una se representa la correlación clínico-histológica de la lesión, tinción con hematoxilina y eosina.

Solo el paciente representado en la figura 5 tuvo $R E$ al tratamiento. Los autores plantean que, a pesar de los excelentes resultados que viene mostrando el uso del HeberPAG ${ }^{\circledR}$, existe la posibilidad de no alcanzar una respuesta clínica, principalmente cuando las características del tumor impiden la correcta difusión del fármaco. Anasagasti, et al. ${ }^{20}$ han registrado resultados impresionantes y muy estimulantes empleando la variante de la combinación de HeberPAG ${ }^{\circledR}$ con quimioterapia y/o radioterapia, pero esta no es objetivo de nuestro trabajo sin que ello implique razones éticas.

Recientemente fue aprobado el uso de vismodegib para el tratamiento del CBC avanzado, este actúa mediante un mecanismo de inhibición de la vía Hedgehog $^{8,32}$.García Vega comparó la eficacia de vismodegib vs. HeberPAG ${ }^{\circledR}$ en el tratamiento del cáncer de piel no melanoma, y encontró un efecto clínico evidentemente mayor con HeberPAG ${ }^{\circledR}$, con una duración de la respuesta superior $8,17,33,34$. 


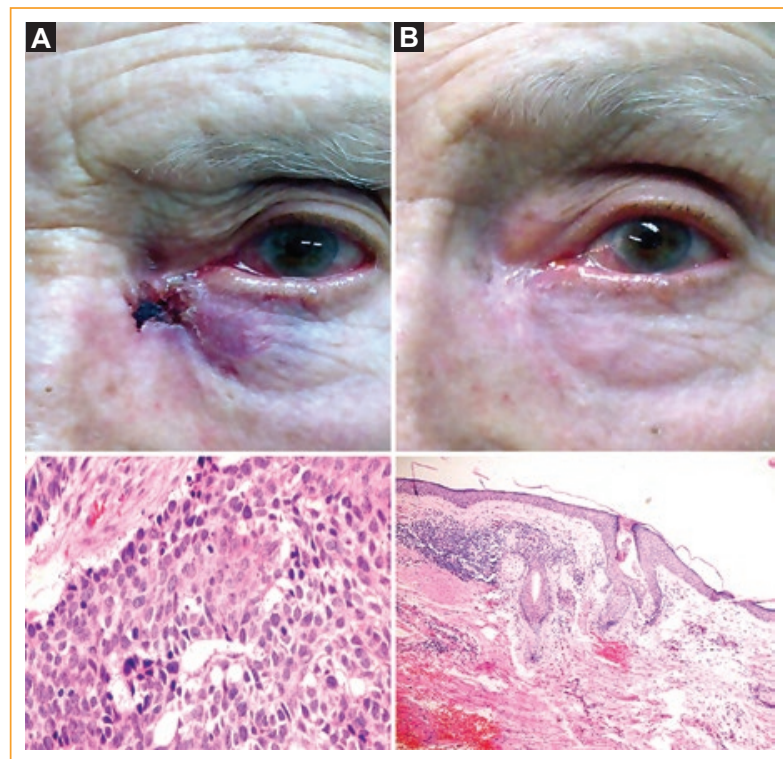

Figura 3. Paciente de 83 años con diagnóstico de CBC, lesión amplia y profunda localizada en el párpado inferior izquierdo, adyacente a la vía lagrimal excretora. En este caso sería muy difícil quirúrgicamente obtener la exéresis total del tumor hacia la profundidad, además la mutilación de las vías lagrimales es inevitable. Se obtuvo respuesta completa, resultado estético satisfactorio y funcionamiento correcto de la vía lagrimal excretora. A: antes del tratamiento; $B$ : después del tratamiento.

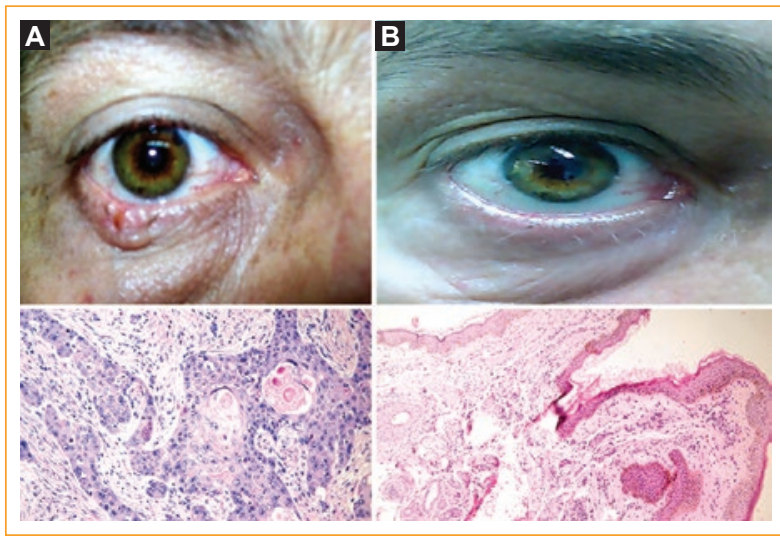

Figura 4. Paciente con CBC tratado previamente con cirugía, lesión que ocupa $1 / 3$ medio de párpado inferior y se extiende hasta el borde libre. Se obtuvo remisión completa. A: antes del tratamiento. B: después del tratamiento.

Los posibles mecanismos involucrados en los efectos clínicos observados con la combinación de IFN pudieran ser los siguientes: el IFN intralesional inicia la

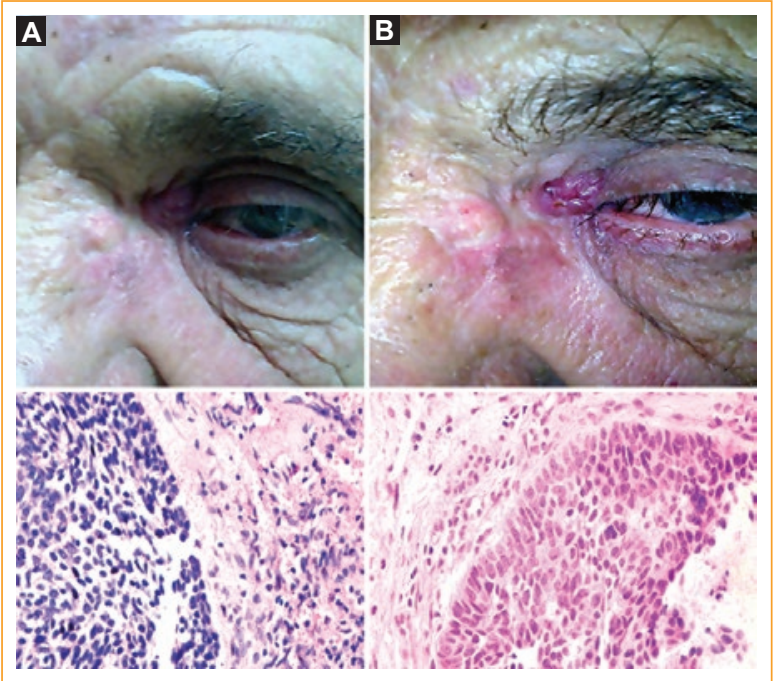

Figura 5. Paciente con CBC localizado en el canto interno izquierdo, sin tratamiento previo. Se obtuvo RE a la semana 16, se realizó cirugía + reconstrucción. Los bordes de sección quirúrgica fueron libres de lesión. A: antes del tratamiento; B: después del tratamiento.

apoptosis de las células de CBC a través de la interacción ligando-receptor CD95, un mecanismo que puede ser reforzado por el IFN- por aumento del receptor CD95. El IFN-, estimulando la expresión del receptor de IFN-, podría contribuir a revertir los bajos niveles observados de este receptor de membrana en las células de CBC. Adicionalmente se ha demostrado que en presencia de IFN-, la señalización intracelular de IFN- es más fuerte. Ambos IFN tienen actividad antiangiogénica y reprimen significativamente la expresión del gen CXCR-4 que puede disminuir la vascularidad que rodea al cáncer de piel no melanoma e impactar en la migración inducida por SDF-1 y en la migración de las células positivas para CXCR-4, como ha sido demostrado en los carcinomas escamosos de cabeza y cuello para IFN-. Todas estas propiedades de los IFN, entre otras, probablemente contribuyen a aumentar la acción antitumoral de esta nueva formulación de IFN ${ }^{20,35}$.

Los eventos adversos más frecuentes con el tratamiento fueron eritema perilesional $(100 \%)$, fiebre (71.4\%), astenia, anorexia, escalofríos (57.1\%) y, en menor frecuencia, náuseas, vómitos y dolor muscular. No se registraron cambios en exámenes hematológicos ni bioquímicos. Resultados que coinciden con otros estudios ${ }^{17,20}$. Todos los eventos notificados fueron de intensidad leve y respondieron bien al tratamiento sintomático, sin necesidad de abandonar la aplicación de HeberPAG ${ }^{\circledR}$. Esto indica que la administración 
sinérgica de IFN alcanza un perfil de seguridad similar a otras presentaciones farmacéuticas de IFN en el mercado, lo que, unido a los efectos clínicos de la combinación de IFN, sugiere que la misma es segura y que es posible su empleo en diseños terapéuticos similares y esquemas de tratamiento prolongados, con el propósito de ofrecer a estos pacientes una opción terapéutica eficaz y segura ${ }^{20}$.

Los efectos adversos reportados en pacientes con vismodegib superaron el $30 \%$ de los casos, e incluyeron espasmo muscular, alopecia, alteraciones del gusto, pérdida de peso y fatiga, y llegaron a ser serios en el $25 \%$ de ellos ${ }^{33}$. Sin embargo, el HeberPAG ${ }^{\circledR}$ se considera un fármaco seguro, con reacciones adversas transitorias, como síntomas catarrales, que pueden ser combatidos con tratamiento medicamentoso previo sin necesidad de abandonar la aplicación de IFN ${ }^{17}$. En una investigación reciente, García Vega, et al. ${ }^{36}$ realizaron un estudio compasivo en una pequeña serie de pacientes con gliomas cerebrales de alto grado sin criterio quirúrgico, donde señalaron que la combinación de IFN brindó signos de mejoría clínica y en la calidad de vida de estos pacientes, con un perfil de seguridad aceptable.

La cirugía micrográfica de Mohs continúa siendo la opción estándar para la cura de todos los CBC localizados en la cara, con una tasa de recurrencia aproximada de un $6.5 \%$ a los 5 años $^{37}$.Sin embargo, debido al tiempo y a las limitaciones de costo, esta se reserva para casos de alto riesgo de recurrencia ${ }^{24,38,39}$.Por tanto, el tratamiento con HeberPAG ${ }^{\circledR}$ gana utilidad antes del tratamiento quirúrgico en pacientes con trastornos de la coagulación, incluyendo el uso de anticoagulantes. También en casos con riesgo alto de defectos de cicatrización, como en pacientes diabéticos, adultos mayores y en aquellos donde la cirugía o la cicatrización pudiera generar alteraciones funcionales. Un beneficio adicional de esta técnica está dado por la conservación de la integridad de la piel, importante en el reconocimiento clínico de las recurrencias.

El principal objetivo del uso del HeberPAG ${ }^{\circledR}$ en CBC perioculares es la preservación del ojo y las estructuras que lo rodean, así como la conservación de la visión, evitando la cirugía reconstructiva y obteniendo un efecto cosmético satisfactorio.

A pesar de que el impacto de los resultados del tratamiento con HeberPAG ${ }^{\circledR}$ promete convertirlo en una nueva y potente opción terapéutica para los tumores de piel no melanomas, el comportamiento y seguimiento a largo plazo de esta serie se publicará en futuros artículos, y brindará información basada en la evidencia para mostrar la estabilidad y sostenibilidad de la respuesta clínica obtenida en la región periocular. Se tendrá en cuenta la inclusión de tamaños muestrales más representativos en futuras investigaciones.

\section{Conclusiones}

Los resultados de este estudio apuntan a que el empleo del HeberPAG ${ }^{\circledR}$ se podría considerar como una alternativa útil y segura como tratamiento conservador en pacientes con CBC periocular cuando otras terapias no son posibles.

\section{Responsabilidades éticas}

Protección de personas y animales. Los autores declaran que los procedimientos seguidos se conformaron a las normas éticas del comité de experimentación humana responsable y de acuerdo con la Asociación Médica Mundial y la Declaración de Helsinki.

Confidencialidad de los datos. Los autores declaran que han seguido los protocolos de su centro de trabajo sobre la publicación de datos de pacientes.

Derecho a la privacidad y consentimiento informado. Los autores han obtenido el consentimiento informado de los pacientes y/o sujetos referidos en el artículo. Este documento obra en poder del autor de correspondencia.

\section{Financiamiento}

Centro de Ingeniería Genética y Biotecnología (CIGB), La Habana. Ministerio de Salud Pública de Cuba (MINSAP).

\section{Conflicto de intereses}

Los autores declaran no tener ningún conflicto de intereses.

\section{Bibliografía}

1. Lewin JM, Carucci JA. Advances in the management of basal cell carcinoma. F1000Prime Rep. 2015;7:53.

2. Karimkhani C, Boyers LN, Dellavalle RP, Weinstock MA. It's time for 'keratinocyte carcinoma' to replace the term 'nonmelanoma skin cancer'. J Am Acad Dermatol. 2015;72:186-7.

3. Mohan SV, Chang AL. Advanced Basal Cell Carcinoma: Epidemiology and Therapeutic Innovations. Curr Dermatol Rep. 2014;3:40-5.

4. Baxter JM, Patel AN, Varma S. Facial basal cell carcinoma. BMJ. 2012;345:e5342

5. García Martín E, Fernández Tirado FJ. Periocular basal cell carcinoma treatment tendencies. Arch Soc Esp Oftalmol. 2010;85:261-2.

6. Wang $\mathrm{CJ}$, Zhang HN, Wu H. Clinicopathologic features and prognostic factors of malignant eyelid tumors. Int J Ophthalmol. 2013;6(4):442-7.

7. Kale SM, Patil SB, Khare N. Clinicopathological analysis of eyelid malignancies - A review of 85 cases. Indian J Plast Surg. 2012;45(1):22-8.

8. Marzuka AG, Book SE. Basal Cell Carcinoma: Pathogenesis, Epidemiology, Clinical Features, Diagnosis, Histopathology, and Management. Yale J Biol Med. 2015;88(2):167-79. 
9. Gulleth Y, Goldberg N, Silverman RP, Gastman BR. What is the best surgical margin for a Basal cell carcinoma: a meta-analysis of the literature. Plast Reconstr Surg. 2010;126(4):1222-31.

10. Prokosch V, Thanos S, Spaniol K, Stupp T. Long-term outcome after treatment with $5 \%$ topical imiquimod cream in patients with basal cell carcinoma of the eyelids. Graefes Arch Clin Exp Ophthalmol. 2011;249:121-5

11. Fantini F, Greco A, Del Giovane C, Cesinaro AM, Venturini M, Zane C., et al. Photodynamic therapy for basal cell carcinoma: clinical and pathological determinants of response. J Eur Acad Dermatol Venereol. 2011;25(8):896-901.

12. Cosgarea R, Susan M, Crisan M, Senila S. Photodynamic therapy using topical 5-aminolaevulinic acid vs. surgery for basal cell carcinoma. J Eur Acad Dermatol Venereol. 2013;27(8):980-4

13. Arits AH, Mosterd K, Essers BA, Spoorenberg E, Sommer A, De Rooij MJ, et al. Photodynamic therapy versus topical imiquimod versus topical fluorouracil for treatment of superficial basal-cell carcinoma: a single blind, non-inferiority, randomised controlled trial. Lancet Oncol. 2013;14(7):647-54.

14. Smith V, Walton S. Treatment of facial basal cell carcinoma: A review. Hindawi Publishing Corporation. J Skin Can. 2011;2011:380371.

15. Lasudry J. Management of eyelid tumors: general considerations. J Fr Ophtalmol. 2011;34(10):741-54.

16. Good LM, Miller MD, High WA. Intralesional agents in the management of cutaneous malignancy: a review. J Am Acad Dermatol. 2011; 64(2):413-22.

17. Garcia-Vega $Y$, Anasagasti-Angulo L, Valenzuela-Silva C, Navarro-Mestre M, Maribeth-Ordoñez S, Acosta-Medina D, et al. Retrospective Study of Periocular Non Melanoma Skin Cancer Treated with the Combination of IFN alpha2b and Gamma (HeberPAG $\left.{ }^{\circledR}\right)$. J Clin Exp Ophthalmol. 2015;6:478

18. Jiménez Barbán $Y$, Vega Pupo $C$, Vila Pinillo D, Fernández Ychaso G Arias Núñez V, Bello Rivero I. Uso de HeberPAG ${ }^{\circledR}$ en carcinoma basocelular periocular. Rev Cubana Oftalmol. 2014;27:3.

19. Bello-Rivero I, Garcia-Vega Y, Valenzuela-Silva C. Development of a new formulation of interferons (HEBERPAG ${ }^{\circledR}$ ) for BCC treatment. J Cancer Res Ther. 2013;1(10):235-243.

20. Anasagasti Angulo L, Garcia Vega Y, Barcelona Perez S, Lopez Saura P, Bello Rivero I. Treatment of advanced, recurrent, resistant to previous treatments basal and squamous cell skin carcinomas with a synergistic formulation of interferons. Open, prospective study. BMC Cancer. 2009;9:262-74

21. García Vega Y, García García I, Collazo Caballero SE, Santely-Pravia EE, Cruz-Ramírez A, Tuero-Iglesias AD, et al. Pharmacokinetic and pharmacodynamic characterization of a new formulation containing synergistic proportions of interferons alpha-2b and gamma (HeberPAG $\left.{ }^{\circledR}\right)$ in patients with mycosis fungoides: an open-label trial. BMC Pharmacol Toxicol. 2012;13:20.

22. Rene C. Oculoplastic aspects of ocular oncology. Eye. 2013;27:199-207.

23. Hayano SM, Whipple KM, Korn BS, Kikkawa DO. Principles of Periocula Reconstruction following Excision of Cutaneous Malignancy. J Skin Cancer. 2012;2012:438502.

24. Lewin JM, Carucci JA. Advances in the management of basal cell carcinoma. F1000Prime Rep. 2015;7:53.
25. Wu TP, Stein JA. Nonmelanoma skin cancer in young women. J Drugs Dermatol. 2013;12:568-72.

26. Göppner D, Leverkus M. Basal Cell Carcinoma: From the Molecular Understanding of the Pathogenesis to Targeted Therapy of Progressive Disease. J Skin Cancer. 2011;2011:650258.

27. Wehner MR, Shive ML, Chren MM, Han J, Qureshi AA, Linos E. Indoor tanning and non-melanoma skin cancer: systematic review and meta-analysis. BMJ. 2012;345:e5909.

28. Ferrucci LM, Cartmel B, Molinaro AM, Leffell DJ, Bale AE, Mayne ST. Indoor tanning and risk of early-onset basal cell carcinoma. J Am Acad Dermatol. 2012;67(4):552-62.

29. Robinson SN, Zens MS, Perry AE, Spencer SK, Duell EJ, Karagas MR Photosensitizing agents and the risk of non-melanoma skin cancer: a population-based case-control study. J Invest Dermatol. 2013; 133(8):1950-5.

30. Stern RS. PUVA Follow-Up Study. The risk of squamous cell and basal cell cancer associated with psoralen and ultraviolet A therapy: a 30-year prospective study. J Am Acad Dermatol. 2012:66(4):553-62.

31. Danial C, Lingala B, Balise R, Oro AE, Reddy S, Colevas A, et al. Markedly improved overall survival in 10 consecutive patients with metastatic basal cell carcinoma. Br J Dermatol. 2013;169:673-6.

32. Von Hoff DD, LoRusso PM, Rudin CM, Reddy JC, Yauch RL, et al. Inhibition of the hedgehog pathway in advanced basal-cell carcinoma. N Engl J Med. 2009:361: 1164-72.

33. Sekulic A, Migden MR, Oro AE, Dirix L, Lewis KD, Hainsworth JD, et al Efficacy and safety of vismodegib in advanced basal-cell carcinoma. N Engl J Med. 2012;366:2171-9.

34. Chang AL, Solomon JA, Hainsworth JD, Goldberg L, McKenna E, Day BM, et al. Expanded access study of patients with advanced basal cell carcinoma treated with the Hedgehog pathway inhibitor, vismodegib. J Am Acad Dermatol. 2014;70(1):60-9.

35. Bello C, Vazquez-Blomquist D, Miranda J, Garcia Y, Novoa LI, Palenzuela $D$, et al. Regulation by IFN- $\alpha /$ IFN- $\gamma$ Co-Formulation (HerberPAG ${ }^{\circledR}$ ) of Genes Involved in Interferon-STAT-Pathways and Apoptosis in U87MG. Curr Top Med Chem. 2014;14(3):351-8.

36. Garcia Vega $Y$, Salva Camaño S, García Iglesias E, Cubero-Rego D, González-Gonzalez J, Bello-Rivero I. CIGB-128, as compassionate intracranial treatment in patients with non-operable or progressive high grade gliomas. J Cancer Res Ther. 2015;3(11):136-43.

37. Smeets NW, Kuijpers DI, Nelemans P, Ostertag JU, Verhaegh ME, et al. Mohs' micrographic surgery for treatment of basal cell carcinoma of the face-results of a retrospective study and review of the literature. $\mathrm{Br} \mathrm{J}$ Dermatol. 2004;151:141-7.

38. Takamori S, Kong K, Varma S, Leach I, Williams HC, Notingher I. Optimization of multimodal spectral imaging for assessment of resection margins during Mohs micrographic surgery for basal cell carcinoma. Biomed Opt Express. 2015;6:98-111.

39. Chren MM, Linos E, Torres JS, Stuart SE, Parvataneni R, Boscardin WJ. Tumor recurrence 5 years after treatment of cutaneous basal cell carcinoma and squamous cell carcinoma. J Invest Dermatol. 2013; 133(5):1188-96. 